\title{
Generation of maximally entangled states and coherent control in quantum dot microlenses ${ }^{*}$
}

Cite as: Appl. Phys. Lett. 112, 153107 (2018); https://doi.org/10.1063/1.5020242

Submitted: 21 December 2017 . Accepted: 17 March 2018 . Published Online: 12 April 2018

Samir Bounouar, Christoph de la Haye, Max Strauß, Peter Schnauber (D), Alexander Thoma, Manuel Gschrey, Jan-Hindrik Schulze, André Strittmatter, Sven Rodt, and Stephan Reitzenstein (D)

\section{COLLECTIONS}

A This paper was selected as an Editor's Pick
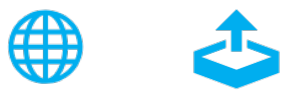

\section{ARTICLES YOU MAY BE INTERESTED IN}

On-demand generation of background-free single photons from a solid-state source Applied Physics Letters 112, 093106 (2018); https://doi.org/10.1063/1.5020038

Polarization-entangled photons from an InGaAs-based quantum dot emitting in the telecom C-band

Applied Physics Letters 111, 133106 (2017); https://doi.org/10.1063/1.4994145

Visualization of anomalous Ettingshausen effect in a ferromagnetic film: Direct evidence of different symmetry from spin Peltier effect

Applied Physics Letters 112, 152403 (2018); https://doi.org/10.1063/1.5022759

Lock-in Amplifiers Find out more today

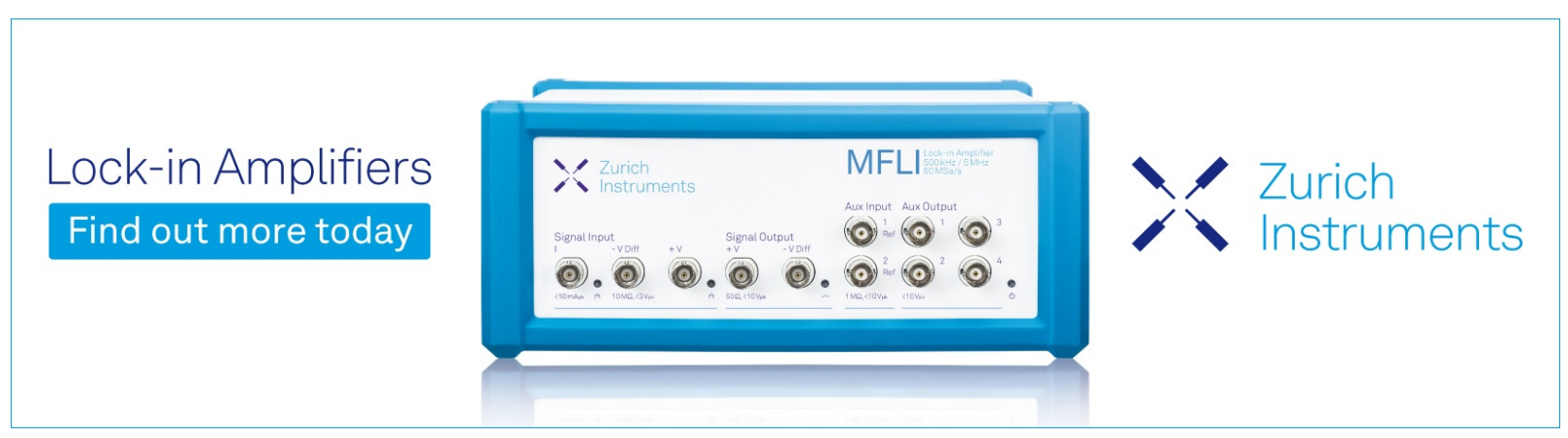




\title{
Generation of maximally entangled states and coherent control in quantum dot microlenses
}

\author{
Samir Bounouar, ${ }^{\text {a) }}$ Christoph de la Haye, Max Strauß, Peter Schnauber, Alexander Thoma, \\ Manuel Gschrey, Jan-Hindrik Schulze, André Strittmatter, ${ }^{\text {b) }}$ Sven Rodt, \\ and Stephan Reitzenstein \\ Institut für Festkörperphysik, Technische Universität Berlin, 10623 Berlin, Germany
}

(Received 21 December 2017; accepted 17 March 2018; published online 12 April 2018)

\begin{abstract}
The integration of entangled photon emitters in nanophotonic structures designed for the broadband enhancement of photon extraction is a major challenge for quantum information technologies. We study the potential of quantum dot (QD) microlenses as efficient emitters of maximally entangled photons. For this purpose, we perform quantum tomography measurements on InGaAs QDs integrated deterministically into microlenses. Even though the studied QDs show non-zero excitonic fine-structure splitting (FSS), polarization entanglement can be prepared with a fidelity close to unity. The quality of the measured entanglement is only dependent on the temporal resolution of the applied single-photon detectors compared to the period of the excitonic phase precession imposed by the FSS. Interestingly, entanglement is kept along the full excitonic wave-packet and is not affected by decoherence. Furthermore, coherent control of the upper biexcitonic state is demonstrated. Published by AIP Publishing. https://doi.org/10.1063/1.5020242
\end{abstract}

Most photonic quantum computation ${ }^{1}$ and quantum communication protocols ${ }^{2}$ rely on the availability of highly entangled photon pairs. Moreover, entanglement plays a pivotal role in linking the nodes of quantum networks. ${ }^{3}$ In this context, the excitonic-biexcitonic radiative cascade in quantum dots (QDs) has proved to be a very promising candidate for the generation of polarization entangled photon pairs, in particular because of the triggered emission of photons. ${ }^{4,5}$ The main obstacle encountered in the generation of entangled photon pairs with QDs has been the finite excitonic finestructure splitting (FSS), giving a "which-path" information on the exciton-biexciton radiative cascade. ${ }^{6}$ In recent years, various growth and post-growth techniques have been developed to reduce the FSS. Efforts were made in approaches as diverse as epitaxial growth in the (111) direction, ${ }^{8}$ growth of highly symmetric GaAs QDs, ${ }^{9-13}$ rapid thermal annealing, ${ }^{7}$ external piezo-applied stress, ${ }^{14}$ or electric ${ }^{15}$ and magnetic field tuning. ${ }^{16,17}$ Despite some of them being successful, they are technologically demanding and can negatively affect the quality of the emission, reducing the quantum efficiency ${ }^{18}$ and the spin coherence. ${ }^{15}$ A second issue is the efficient broadband extraction of entangled pairs emitted by a semiconductor QD located in the high refractive index host material. While cavity enhanced emission of entangled photon pairs was achieved using a QD coupled to narrow-band hybridized micropillar cavity modes, ${ }^{19}$ the scalability and the reproducibility of this very demanding concept are still nonsolved issues. Since the spectral separation between the excitonic and the biexcitonic transitions, namely, the biexciton binding energy, is typically in the range of a few meV, solutions featuring broadband enhancement of photon extraction are most suitable for the realization of QD based entangled

\footnotetext{
${ }^{\text {a) }}$ Author to whom correspondence should be addressed: samir.bounouar@ tu-berlin.de

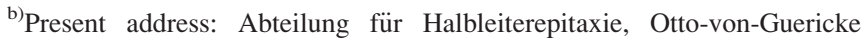
Universität, 39106 Magdeburg, Germany.
}

photon-pair sources. Over the last decade, a few approaches including photonic wires and microlenses ${ }^{20-22}$ tackled this challenge and are good candidates for the production of entangled photon pairs.

We present here a study on single semiconductor QDs integrated deterministically into microlenses. ${ }^{22}$ Since these structures allow for a broadband extraction of the excitonic (X) and biexcitonic (XX) photons as well as for enhanced focusing of the resonant laser, ${ }^{23}$ they are very interesting structures for applications in the field of photonic quantum information technology. We show that two key requirements are fulfilled by these nanostructures. First, by applying pulsed resonant two-photon excitation of the biexciton, we show that the quantum dot upper-state can be coherently addressed and controlled. Second, time resolved quantum tomography is performed on photon pairs emitted by the radiative $\mathrm{XX}-\mathrm{X}$ cascade of the QD. We take advantage of Heisenberg's relation, expressing that a higher temporal resolution in determining the dynamics of the $\mathrm{XX}-\mathrm{X}$ decay implies larger uncertainty in energy which can be larger than the related excitonic fine structure of the QD. In this situation, the "which-path information" is lost and quantum entanglement of the paired photons can be measured even in the presence of a FSS. The observed degree of entanglement is actually solely limited by the detectors' temporal resolution which has to be compared with the inverse precession frequency of the excitonic phase imposed by the FSS. ${ }^{24}$ In this work, we discuss the efficiency of the entangled pair photon emission, the evolution of the two-photon state, and in particular, the conservation of the entanglement quality along the whole wavepacket. By performing the tomography on two different QDs with different FSS, we demonstrate the reproducibility of the method and discuss the effect of the FSS with respect to the setup resolution and its impact on the measured entanglement quality. The latter is also measured as a function of the applied time post-selection. 
Our experiments are carried out on self-assembled InGaAs/GaAs QDs grown by metal-organic chemical vapor deposition. The QDs are integrated into microlenses with a backside distributed Bragg reflector by 3D in-situ electronbeam lithography. ${ }^{22}$ A scanning electron microscopy (SEM) image of such a device is displayed in the inset of Fig. 1(c). Two different QD-microlenses denoted as QDM1 and QDM2 are studied in the following. Figure 1(a) shows $\mu \mathrm{PL}$ spectra of QDM1 and QDM2 under non-resonant excitation at $532 \mathrm{~nm}$. Two emission lines in each spectrum are identified as $\mathrm{X}$ and XX transitions of the target QD. The inset of Fig. 1(a) (right panel inset) shows the typical emission spectrum of a QDmicrolens under resonant pulsed two-photon excitation of the biexciton. Figure 1(c) presents the power dependence of the $\mathrm{X}$ and $\mathrm{XX}$ intensities [as measured by a single-photon counting module (SPCM)] as a function of the applied pulse area. The laser is placed at an energy resonant to the virtual state, enabling the two-photon excitation of the biexciton. This excitation scheme has become a well-established and powerful technique $e^{5,24,25}$ and is nowadays considered as a critical prerequisite for the coherent generation of entangled photons by QDs. We perform these measurements in confocal configuration with a resonant excitation setup. Typical Rabi oscillations of the biexcitonic and excitonic line intensities are observed when the laser pulse area is increased, accounting for the coherent control of the QD biexcitonic state in the Blochsphere. ${ }^{27}$ The first maximum of the curves plotted in Fig. 1(c) represents the first inversion of the biexcitonic population $(\pi$ pulse) and the ideal operation point of the source. The FSS of QDM1 and QDM2 is determined by polarization-dependent



FIG. 1. (a) $\mu$ PL spectra of QDM1 (left panel) and QDM2 (right panel) under non-resonant excitation (left panel inset: relative energy of the $\mathrm{X}$ and $\mathrm{XX}$ lines as a function of the detection polarization angle for QDM1 and QDM2. FSS values of $16 \mu \mathrm{eV}$ for QDM1 and $30 \mu \mathrm{eV}$ for QDM2 are determined by a sinusoidal fit of the experimental data). Right panel inset: exemplary $\mu \mathrm{PL}$ spectrum of a QD-microlens under resonant two-photon excitation. (b) Scheme of the relevant states in a QD for the generation of entangled photon pairs. (c) $\mu \mathrm{PL}$ intensity of the $\mathrm{XX}$ transition as a function of the two-photon resonant pulse area. (d) Scanning Electron Microscopy image of a deterministically fabricated QD-microlens. micro-photoluminescence ( $\mu \mathrm{PL}$ ) spectroscopy. The inset of Fig. 1(a) shows the relative energies of $X$ (blue curve) and $\mathrm{XX}$ (red curve) as a function of the detection angle in linear polarization. Sinusoidal fits to the experimental data yield a FSS of $(15 \pm 1) \mu \mathrm{eV}$ for QDM1 and $(30 \pm 1) \mu \mathrm{eV}$ for QDM2, respectively.

QDM1 shows a single-photon extraction efficiency of 8.4 $(+/-1) \%$, which leads to a photon pair efficiency (X and XX) of $0.71(+/-0.02) \%$. These values are comparable to the actual state of the art in the field of efficient entanglement production with semi-conductor devices. ${ }^{8,26}$ The achieved value is about two orders of magnitude higher than the values reported for standard self-assembled quantum dots. ${ }^{10,13,28}$ QDM2 shows a single-photon extraction efficiency of $6(+/-1) \%$ [photon-pair efficiency of $0.36(+/-0.02) \%]$. The measurement method of the extraction efficiency is based on a previous work ${ }^{22}$ and is provided in the supplementary material.

In QDs with a non-zero FSS, the spin up and spin down exciton states [labelled $\left|X_{H}\right\rangle$ and $\left|X_{V}\right\rangle$ in Fig. 1(b), respectively] are non-degenerate and are no eigenstates of the system. The exciton state will therefore evolve with time. One can write the resulting two-photon state as follows: ${ }^{18}$

$$
|\psi(\tau)\rangle=\frac{1}{\sqrt{2}}\left(|H H\rangle+e^{i \Delta \tau}|V V\rangle\right),
$$

with $\Delta$ being the frequency corresponding to the FSS energy, $\mathrm{HH}$ (VV) describing the polarization of the two photons being horizontal (vertical), and $\tau$ the time delay between the excitonic and biexcitonic photons. This state can be rewritten in the diagonal basis (D/A) to show that it oscillates between $\left|\phi^{+}\right\rangle=\frac{1}{\sqrt{2}}(|D D\rangle+|A A\rangle)$ and $\left|\phi^{-}\right\rangle=\frac{1}{\sqrt{2}}(|D A\rangle+|A D\rangle)$. One obtains a similar result in the circular basis $(\mathrm{R} / \mathrm{L})$. This means that the excitonic phase evolution, which leads to this oscillation, can be tracked by correlating the photons when they are projected in these two bases.

For the quantum tomography measurements, we use a time resolved polarization-dependent cross-correlation setup. Photons from the coherently driven (under $\pi$-pulse conditions) XX-X cascade are polarization-projected in the $3 \mathrm{com}-$ plementary bases $(\mathrm{H} / \mathrm{V}, \mathrm{D} / \mathrm{A}$ and $\mathrm{R} / \mathrm{L})$, and the coincidence rate is measured as a function of the delay between the $\mathrm{XX}$ photons and the $\mathrm{X}$ photons. The 16 measurements necessary for the full quantum tomography and the corresponding normalized correlation functions are plotted in Fig. 2. The observed time dependent oscillations due to the excitonic phase evolution are discussed above and occur when both photons are projected in the right circular polarization $\mathrm{R}$ or in the diagonal polarization $\mathrm{D}$. On the other hand, the measurements in the linear basis (HH and HV), showing no oscillation, exhibit classical correlations. The temporal resolution of the setup is estimated to be 100 ps (full width at half maximum), and each coincidence time bin is $4 \mathrm{ps}$.

Based on the experimental data presented in Fig. 2, the density matrices of the generated two-photon states are reconstructed using a maximum-likelihood estimation. Figure 3 shows the reconstructed density matrices obtained for the first maximum (Fig. 2, red line) and the first minimum (Fig. 2, green line) observed in the DD curve of Fig. 2 (red line marked). Figure 3(a) shows the state of the QD directly after the emission of the biexcitonic photon (left panel for QDM1 


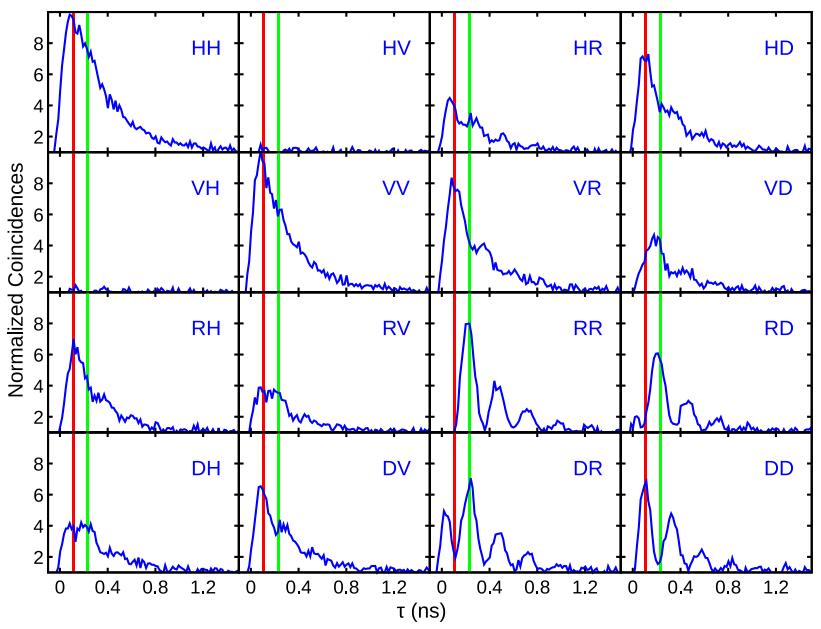

FIG. 2. 16 time resolved polarization-dependent correlation measurements used for the quantum tomography for QDM1. The red (green) line represents the time bin used for the density matrix reconstruction denoted as $\rho_{1}\left(\rho_{2}\right)$ in the following.

and right panel for QDM2). For the sake of comparison, the ideal density matrix (real part and imaginary part) of $\left|\phi^{+}\right\rangle$between the experimental density matrices for QDM1 and QDM2 is displayed in Fig. 3(a). The fidelities $\left\{F\left(\phi^{+}\right)=\operatorname{Tr}\left[{\sqrt{\sqrt{\rho_{1}} \rho\left(\phi^{+}\right) \sqrt{\rho_{1}}}}^{2}\right\}\right.$ of the experimental density matrix $\rho_{1}$ to $\left|\phi^{+}\right\rangle$are estimated to be $0.73 \pm 0.03$ for QDM1 and $0.69 \pm 0.04$ for QDM2. Since the phase in QDM1 is evolving slower than that in QDM2, the setup is able to better resolve the oscillation for QDM1 which also shows a higher degree of entanglement. At longer delays, the QD state rotates towards $\left|\phi^{-}\right\rangle$. Figure 3(b) shows the reconstructed density matrices obtained for the first minimum of the DD coincidence curve (green line marked in Fig. 2). They resemble the $\left|\phi^{-}\right\rangle$state [presented in the inset of Fig. 3(b)]. $F\left(\phi^{-}\right)$values are estimated to be $0.80 \pm 0.03$ for QDM1 and $0.68 \pm 0.04$ for QDM2. The presence of non-zero offdiagonal elements in the imaginary part of the reconstructed density matrix is attributed to the convolution to the setup response, which leads to mixing of the phase of the evolving two-photon state. Additionally, they could also be a consequence of the non-perfect preparation of the tomography states, especially in the circular basis.

Figure 4(a) depicts the time evolution of the fidelity to the two Bell states, $F\left(\phi^{+}\right)$and $F\left(\phi^{-}\right)$, of the two-photon state. The top panel depicts the exciton decay curve (the photons are not polarization-projected) and is shown here as a reference. As expected, because of the excitonic phase evolution, the entangled two-photon state evolves between $\left|\phi^{+}\right\rangle$and $\left|\phi^{-}\right\rangle$. This curve is obtained after reconstructing the density matrices for each delay bin (of 4 ps width) and evaluating their fidelity to the $\left|\phi^{+}\right\rangle$state (blue) and to the $\left|\phi^{-}\right\rangle$state. Interestingly, the oscillations can be clearly observed along the whole exciton wavepacket [plotted in the top panel of Fig. 4(a)], indicating that the entanglement of the QD state is mostly unaffected by decoherence. Figure 4(c) shows $F\left(\phi^{+}\right)$ and $F\left(\phi^{-}\right)$as a function of the time windows used for the tomography. The fidelities for both quantum dots are decreasing quickly as the time window is enlarged, and for a postselection exceeding $20 \%$ of the total exciton wavepacket, no entanglement can be observed any more. An optimal working point is obtained by post-selection of around $15 \%$ of the excitonic photons, presenting a good compromise between fidelity to the Bell state and the photon count rate.

In order to evaluate the actual quality of the entanglement between the photons emitted by the QD, we deconvoluted the data from the time response of the experimental tomography setup with a temporal resolution of $100 \mathrm{ps}$. The theoretical polarization-dependent cross-correlation function $^{24}$ is convoluted to a Gaussian curve (100 ps full width at half maximum), as measured from the setup response. The resulting curve is fitted to the 16 tomography data curves. All the function parameters, such as the polarization angles, the FSS frequency, and the decay time, were obtained from the experiment and are kept constant. Only a multiplying factor and an offset are left as free parameters for the data fitting (see supplementary material). The deconvoluted fit function is used in order to reconstruct the new density
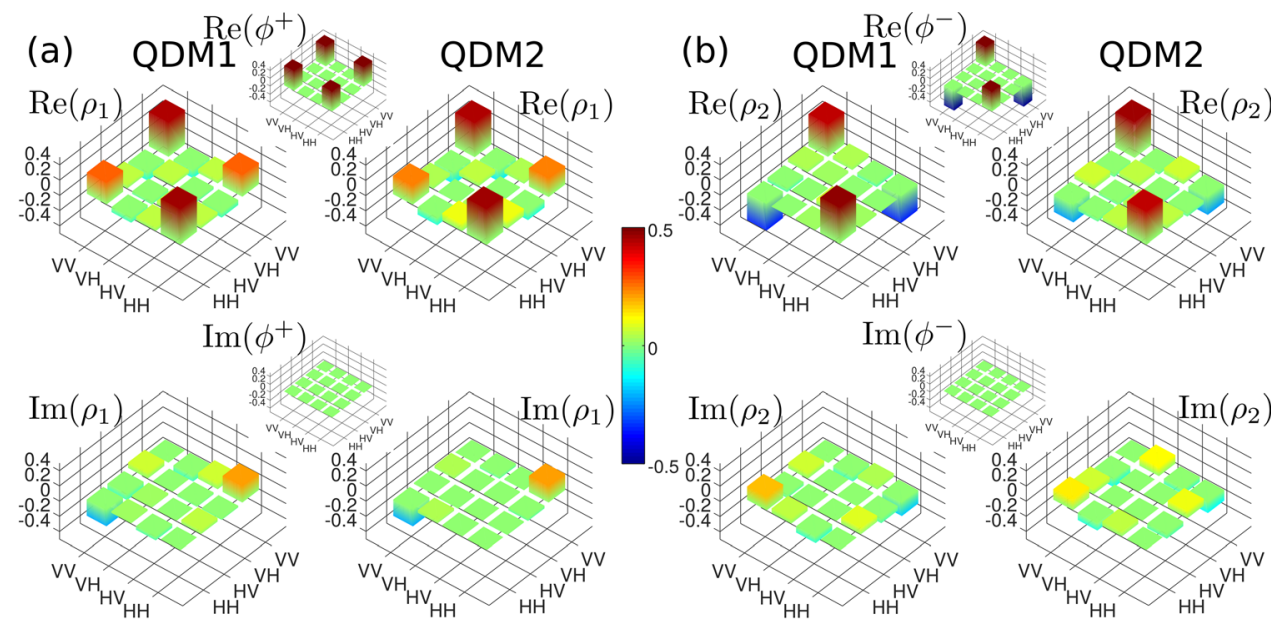

FIG. 3. (a) Density matrices reconstructed for a delay corresponding to the first maximum of the DD coincidence curve (denoted as $\rho_{1}$, 4 ps selection time window), for QDM1 (left panel) and for QDM2 (right panel). Real parts are displayed on the top and imaginary parts on the bottom part of the graph. Theoretical real parts and imaginary parts of the maximally entangled state $\left|\phi^{+}\right\rangle$are plotted between the matrices of QDM1 and QDM2, as a reference. (b) The same density matrices reconstructed for a delay corresponding to the first minimum of the DD coincidence curve (denoted as $\rho_{2}$, 4 ps selection time window). Theoretical real parts and imaginary parts of the maximally entangled state $\left|\phi^{-}\right\rangle$are plotted between the matrices of QDM1 and QDM2, as a reference. 
(a)

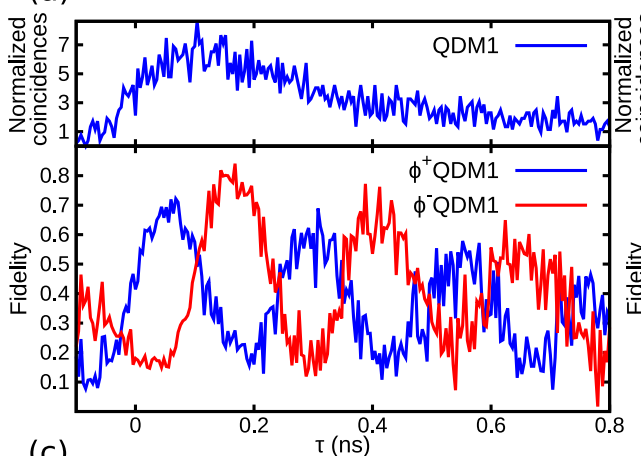

$$
\text { (c) }
$$



(b)


FIG. 4. (a) Data obtained for QDM1: (upper panel) Exciton decay curve. (lower panel) Measured fidelities to the maximally entangled Bell states (blue curve for $\left|\phi^{+}\right\rangle$and red curve for $\left.\left|\phi^{-}\right\rangle\right)$. (b) The same data obtained with QDM2. Measured fidelity as a function of the percentage of the post-selected excitonic wavepacket (c) for QDM1 and (d) for QDM2. The red curves in (c) and (d) represent the fidelities measured as a function of the wave-packet percentage, when the QDs are in the $\left|\phi^{+}\right\rangle$state (i.e., red cursor in Fig. 2, first maximum of the DD curve). The blue curves represent the same measurements when the QDs are in the $\left|\phi^{-}\right\rangle$state (i.e., green cursor in Fig. 2, first minimum of the DD curve). matrices representing the emitted two-photon states without the effect of the experimental resolution. Figure 5 shows the results obtained from the quantum tomography after deconvolution. Figure 5(a) shows the decay curve (upper panel), the fidelity to the Bell states (middle panel), and the negativity (lower panel) for QDM1. The fidelities are showing oscillations with an amplitude very close to unity without damping along the full wavepacket, showing that QDM1 emits nearly perfectly entangled photons. This is confirmed by the negativity (lower panel), quantifying the separability of the density matrix. A value close to 0.5 (maximal entanglement) is found up to a delay of $0.8 \mathrm{~ns}$.
Figure 5(b) shows very similar results for QDM2 with larger FSS. For instance, oscillations with close to unity amplitude can also be observed in the fidelity curves. For this QD, more pronounced damping of the oscillations is observed, indicating that the quality of the entanglement is significantly reduced for delays larger than $0.5 \mathrm{~ns}$. The decrease in the negativity with respect to the delay is consistent with this observation. Even if simultaneous jumps of the excitonic and biexcitonic phases do not affect the QD entanglement, cross-dephasing processes, ${ }^{18}$ such as exciton spin "flip-flop" processes, could be the reason for such a degradation of the entanglement quality for this particular QD. The
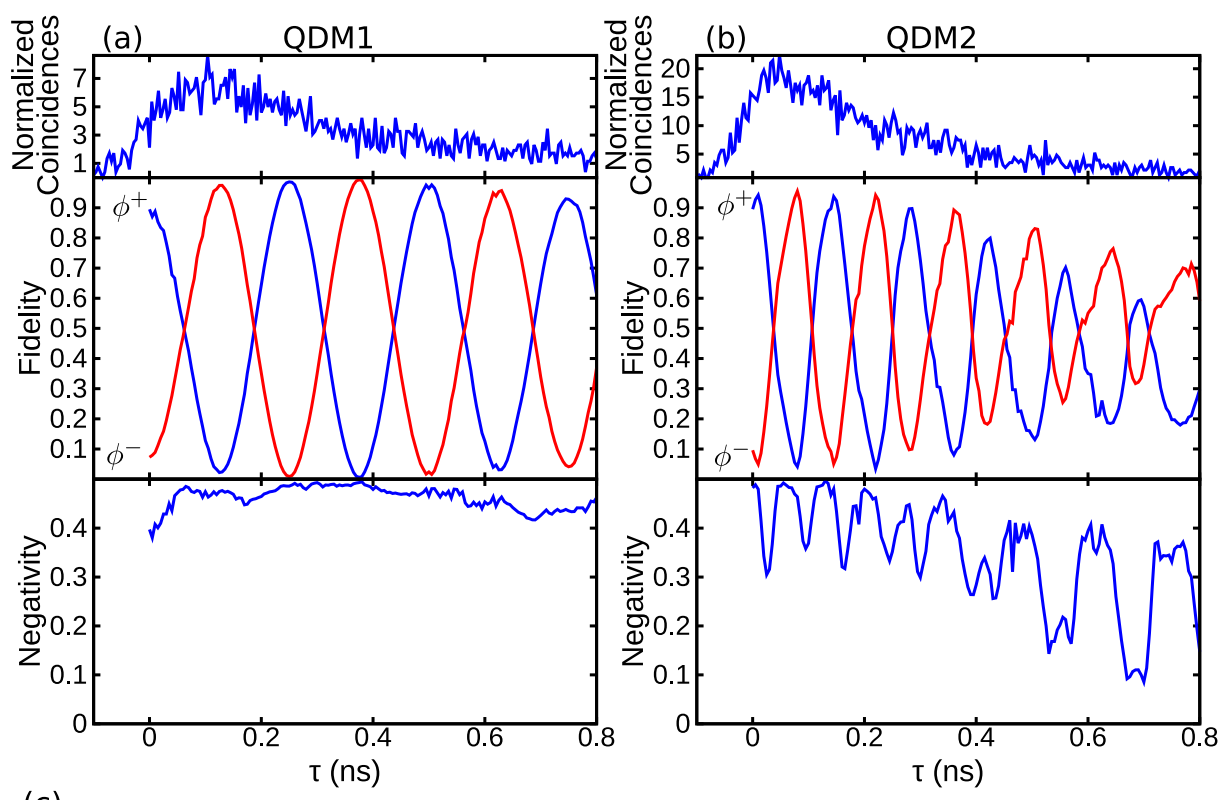

FIG. 5. (a) Deconvoluted data obtained for QDM1. Upper panel: Exciton decay curve. Intermediate panel: Measured fidelities to the maximally entangled Bell states (blue curve for $\left|\phi^{+}\right\rangle$and red curve for $\left.\left|\phi^{-}\right\rangle\right)$. Lower panel: Negativity of the two-photon state as a function of the delay after deconvolution, (b) Deconvoluted data obtained with QDM2, and (c) Superposed norms of the density matrices reconstructed before convolution (in color) and corrected after deconvolution (in blue) for

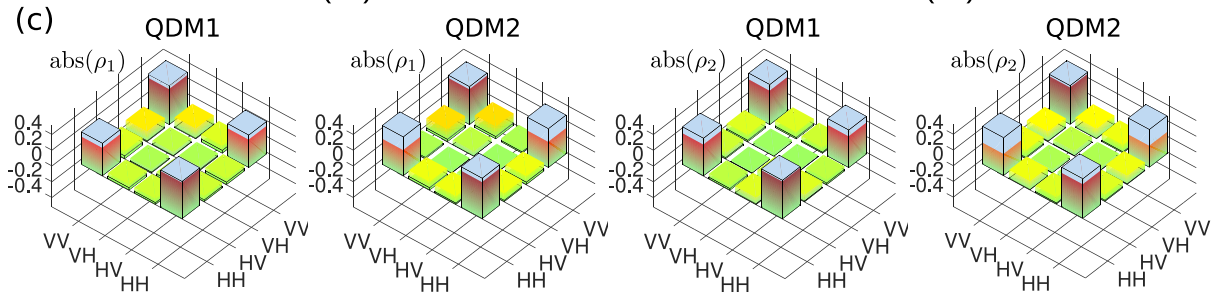
QDM1 and QDM2. 
importance of these processes can be different from dot to dot since they are phonon mediated and the exciton-phonon coupling strength is strongly influenced by the size of the dot. This could explain why it affects QDM2 more strongly than QDM1. Moreover, in the case of QDM2, the deconvolution is not able to fully suppress the periodic drops of the negativity. This might be attributed to the less accurate preparation of the circular state in one arm of the tomography setup, which, even corrected during the tomography, can be seen on the negativity.

In conclusion, we have shown that deterministically fabricated QD-microlenses with broadband photon extraction are very suitable for the reliable generation of entangled photon pairs. This is demonstrated by two-photon excitation of the biexciton in QD-microlenses, where for finite FSS, the entanglement fidelity is only limited by the experimental time resolution. Interestingly, the decoherence of $\mathrm{XX}$ and $\mathrm{X}$ is not affecting their fidelity to the Bell states. These achievements open the possibility of using QDs, showing FSS exceeding $10 \mu \mathrm{eV}$ in photonic quantum technology schemes, but at the price of an event "post-selection." Our experiments show that after temporal post-selection, quantum dot microlenses can compete with the entanglement quality of spontaneous parametric down conversion-based sources working at low pumping powers, ${ }^{29}$ even if our technology is not yet competitive against their emission efficiency at higher powers. ${ }^{30}$ In this respect, the microlenses or other high efficiency broadband nanophotonic elements are of great interest. However, a reduction of the FSS is still of great importance since it allows for the use of less narrow post-selection windows, slower detectors, and shorter integration times. Entangled photon pair emission from such optical nanodevices represents a significant step towards the practical and generalized realization of entanglement swapping or teleportation experiments which are key requirements for long-distance quantum communication and photonic quantum computation.

See supplementary material for further details on the quantum tomography measurements, the deconvolution procedure, the errors, the corrections, and the determination of the quantum efficiencies.

The research leading to these results has received funding from the German Research Foundation via Project No. RE2974/12-1, SFB 787, and from the German Federal Ministry of Education and Research (BMBF) through the VIP-Project QSOURCE (Grant No. 03V0630).

${ }^{1}$ E. Knill, R. Laflamme, and G. A. Milburn, Nature 409, 46-52 (2001).

${ }^{2}$ W. Tittel and G. Weihs, Quantum Inf. Comput. 1, 3-56 (2001).

${ }^{3}$ H. J. Kimble, Nature 453, 1023-1030 (2008).
${ }^{4}$ M. Müller, S. Bounouar, K. D. Jöns, M. Glässl, and P. Michler, Nat. Photonics 8, 224-228 (2014).

${ }^{5}$ S. Bounouar, M. Müller, A. M. Barth, M. Glässl, V. M. Axt, and P. Michler, Phys. Rev. B 91, 161302 (2015).

${ }^{6}$ N. Akopian, N. H. Lindner, E. Poem, Y. Berlatzky, J. Avron, D. Gershoni, B. D. Gerardot, and P. M. Petroff, Phys. Rev. Lett. 96, 130501 (2006)

${ }^{7}$ R. J. Young, R. M. Stevenson, A. J. Shields, P. Atkinson, K. Cooper, D. A. Ritchie, K. M. Groom, A. I. Tartakovskii, and M. S. Skolnick, Phys. Rev. B 72, 113305 (2005).

${ }^{8}$ M. A. M. Versteegh, M. E. Reimer, K. D. Jöns, D. Dalacu, P. J. Poole, A. Gulinatti, A. Giudice, and V. Zwiller, Nat. Commun. 5, 5298 (2014).

${ }^{9}$ A. Mohan, M. Felici, P. Gallo, B. Dwir, A. Rudra, J. Faist, and E. Kapon, Nat. Photonics 4, 302-306 (2010).

${ }^{10}$ D. Huber, M. Reindl, Y. Huo, H. Huang, J. S. Wildmann, O. G. Schmidt, A. Rastelli, and R. Trotta, Nat. Commun. 8, 15506 (2017).

${ }^{11}$ T. Kuroda, T. Mano, N. Ha, H. Nakajima, H. Kumano, B. Urbaszek, M. Jo, M. Abbarchi, Y. Sakuma, K. Sakoda, I. Suemune, X. Marie, and T. Amand, Phys. Rev. B 88, 041306(R) (2013).

${ }^{12}$ T. H. Chung, G. Juska, S. T. Moroni, A. Pescaglini, A. Gocalinska, and E. Pelucchi, Nat. Photonics 10, 782-787 (2016).

${ }^{13}$ G. Juska, V. Dimastrodonato, L. O. Mereni, A. Gocalinska, and E. Pelucchi, Nat. Photonics 7, 527-531 (2013).

${ }^{14}$ J. Zhang, J. S. Wildmann, F. Ding, R. Trotta, Y. Huo, E. Zallo, D. Huber, A. Rastelli, and O. G. Schmidt, Nat. Commun. 6, 10067 (2015).

${ }^{15}$ A. J. Bennett, M. A. Pooley, R. M. Stevenson, M. B. Ward, R. B. Patel, A. B. de La Giroday, N. Sköld, I. Farrer, C. A. Nicoll, D. A. Ritchie et al., Nat. Phys. 6, 947 (2010).

${ }^{16}$ R. J. Young, R. M. Stevenson, P. Atkinson, K. Cooper, D. A. Ritchie, and A. J. Shields, New J. Phys. 8, 29 (2006).

${ }^{17}$ R. M. Stevenson, R. J. Young, P. See, D. G. Gevaux, K. Cooper, P. Atkinson, I. Farrer, D. A. Ritchie, and A. J. Shields, Phys. Rev. B 73, 033306 (2006).

${ }^{18}$ R. M. Stevenson, A. J. Hudson, A. J. Bennett, R. J. Young, C. A. Nicoll, D. A. Ritchie, and A. J. Shields, Phys. Rev. Lett. 101, 170501 (2008).

${ }^{19}$ A. Dousse, J. Suffczynski, A. Beveratos, O. Krebs, A. Lemaitre, I. Sagnes, J. Bloch, P. Voisin, and P. Senellart, Nature 466, 217-220 (2010).

${ }^{20}$ M. E. Reimer, G. Bulgarini, N. Akopian, M. Hocevar, M. B. Bavinck, M. A. Verheijen, E. P. A. M. Bakkers, L. P. Kouwenhoven, and V. Zwiller, Nat. Commun. 3, 737 (2012).

${ }^{21}$ J. Claudon, J. Bleuse, N. S. Malik, M. Bazin, P. Jaffrennou, N. Gregersen, C. Sauvan, P. Lalanne, and J.-M. Gérard, Nat. Photonics 4, 174-177 (2010).

${ }^{22}$ M. Gschrey, A. Thoma, P. Schnauber, M. Seifried, R. Schmidt, B. Wohlfeil, L. Krüger, J.-H. Schulze, T. Heindel, S. Burger, F. Schmidt, A. Strittmatter, S. Rodt, and S. Reitzenstein, Nat. Commun. 6, 7662 (2015).

${ }^{23}$ S. Bounouar, M. Strauss, A. Carmele, P. Schnauber, A. Thoma, M. Gschrey, J.-H. Schulze, A. Strittmatter, S. Rodt, A. Knorr, and S. Reitzenstein, Phys. Rev. Lett. 118, 233601 (2017).

${ }^{24}$ R. Winik, D. Cogan, Y. Don, I. Schwartz, L. Gantz, E. R. Schmidgall, N. Livneh, R. Rapaport, E. Buks, and D. Gershoni, Phys. Rev. B 95, 235435 (2017).

${ }^{25}$ H. Jayakumar, A. Predojevć, T. Huber, T. Kauten, G. S. Solomon, and G. Weihs, Phys. Rev. Lett. 110, 135505 (2013).

${ }^{26}$ A. Orieux, A. Eckstein, A. Lemaitre, P. Filloux, I. Favero, G. Leo, T. Coudreau, A. Keller, P. Milman, and S. Ducci, Phys. Rev. Lett. 110, 160502 (2013).

${ }^{27}$ S. Stufler, P. Machnikowski, P. Ester, M. Bichler, V. M. Axt, T. Kuhn, and A. Zrenner, Phys. Rev. B 73(12), 125304 (2006).

${ }^{28}$ R. Trotta, J. S. Wildmann, E. Zallo, O. G. Schmidt, and A. Rastelli, Nano Lett. 14, 3439-3444 (2014).

${ }^{29}$ A. Predojevic, S. Grabher, and G. Weihs, Opt. Express 20, 25022-25029 (2012).

${ }^{30}$ X.-L. Wang, L.-K. Chen, W. L. H.-L. Huang, C. Liu, C. Chen, Y.-H. Luo, Z.-E. Su, D. Wu, Z.-D. Li, H. Lu, Y. Hu, X. Jiang, C.-Z. Peng, L. Li, N.-L. Liu, Y.-A. Chen, C.-Y. Lu, and J.-W. Pan, Phys. Rev. Lett. 117, 210502 (2016). 